OPEN

SUBJECT AREAS:

BIOMECHANICS

BIOMEDICAL ENGINEERING

BIOLOGICAL PHYSICS

NUMERICAL SIMULATIONS

Received

20 May 2014

Accepted

28 July 2014

Published

14 August 2014

Correspondence and requests for materials should be addressed to H.I. (h.ishii@rikkyo.ac.

\title{
Effect of soccer shoe upper on ball behaviour in curve kicks
}

\author{
Hideyuki Ishii' ${ }^{1}$ Yoshihisa Sakurai ${ }^{2} \&$ Takeo Maruyama $^{3}$
}

\begin{abstract}
'Department of Sport and Wellness, Rikkyo University, 1-2-26 Kitano, Niiza-shi, Saitama 352-8558, Japan, ${ }^{2}$ Department of Sports Sciences, Japan Institute of Sports Sciences, 3-15-1 Nishigaoka, Kita-ku, Tokyo 1 15-0056, Japan, ${ }^{3}$ Graduate School of Decision Science and Technology, Tokyo Institute of Technology, W9-4, 2-12-1 Ookayama, Meguro-ku, Tokyo 152-8552, Japan.
\end{abstract}

New soccer shoes have been developed by considering various concepts related to kicking, such as curving a soccer ball. However, the effects of shoes on ball behaviour remain unclear. In this study, by using a finite element simulation, we investigated the factors that affect ball behaviour immediately after impact in a curve kick. Five experienced male university soccer players performed one curve kick. We developed a finite element model of the foot and ball and evaluated the validity of the model by comparing the finite element results for the ball behaviour immediately after impact with the experimental results. The launch angle, ball velocity, and ball rotation in the finite element analysis were all in general agreement with the experimental results. Using the validated finite element model, we simulated the ball behaviour. The simulation results indicated that the larger the foot velocity immediately before impact, the larger the ball velocity and ball rotation. Furthermore, the Young's modulus of the shoe upper and the coefficient of friction between the shoe upper and the ball had little effect on the launch angle, ball velocity, and ball rotation. The results of this study suggest that the shoe upper does not significantly influence ball behaviour.

\footnotetext{
$\mathrm{S}$
} occer is the most popular sport in the world. Soccer shoes are essential for playing soccer. New shoes have been developed by considering various concepts related to kicking, such as curving a soccer ball by applying high spin, kicking a knuckle ball with low spin, and kicking a fast (strong) ball. If a player scores a goal through a free kick, the shoes worn by this player may be featured in the media and capture the limelight. However, there is very little pertinent data to verify whether shoes are the basis of the intended effect; the effects of shoes on ball behaviour remain unclear.

A curve kick is a technique that is frequently used in soccer matches for shots on goal, free kicks, corner kicks, and so on. Goals by curve kicks are commonly scored from free kicks in Fédération Internationale de Football Association (FIFA) world cups, and therefore, the kicking technique is considered one of the important elements that can decide the outcomes of matches.

In a soccer kick, the impact phase is important because mechanical phenomena during impact determine the ball behaviour. However, because of the problem of repeatability in measurements, experiments using human subjects $^{1}$ to evaluate the factors that affect ball behaviour at impact with high accuracy have limitations. From empirical measurements and numerical calculations using theoretical equations based on simple modelling, the relationship between the impact and ball behaviour and the factors that affect the ball behaviour have been previously reported for inste $\mathrm{p}^{2,3}$ as well as side-foot kicks ${ }^{4}$. However, it is difficult to apply such methods to the curve kick technique to analyse the relationship between the impact and the ball behaviour for several reasons, the most important ones include diverse foot postures immediately before impact in curve kicks and a wide range of impact points that vary from the dorsal aspect to the medial aspect of the kicking foot; in other words, the impact patterns are diverse. To evaluate the relationship between the impact and the ball behaviour in curve kicks, the shape of the ball-contact area on the foot must be considered.

Finite element analysis is an effective approach for solving this problem. Asai et al..$^{5-7}$ used finite element analysis to investigate the phenomena occurring during impact and the subsequent ball behaviour of a curve kick. Price et al. ${ }^{8-11}$ and Rezaei et al. ${ }^{12}$ developed a finite element ball model to simulate bounce behaviour. However, no studies have been conducted on assessing the validity of the finite element model by comparing the finite element results with the experimental values to simulate the ball behaviour in three dimensions, including launch angle, ball velocity, and ball rotation. A three-dimensional (3D) simulation of ball behaviour would help improve not only player skills but also future product development of soccer shoes and soccer balls, both of which would be very useful. 
Effective models are simple and can solve the research problem. For finite element analysis, it is important that a model can solve the problem without being overly complex, such as the foot model developed by Dai et al. ${ }^{13}$ to assess the effect of socks on walking. The shape of the ball-contact area on the foot must be considered when developing a finite element foot model to evaluate the relationship between the impact and the ball behaviour in curve kicks; however, there is no need to consider in detail the inner structures such as bone shape and ligaments, which helps to simplify the model to some degree.

This study developed a simplified finite element model of the foot and ball to simulate the 3D ball behaviour (launch angle of ball, ball velocity, and ball rotation) caused by the impact of a curve kick and evaluated the validity of the model by comparing the results of the finite element analysis with the empirical results. This study aimed to investigate the factors that affect the ball behaviour in curve kicks by using a validated finite element model and by simulating the ball behaviour for curve kicks by varying the foot velocity immediately before impact, the Young's modulus of the shoe upper, and the coefficient of friction between the shoe upper and the ball.

\section{Results}

Finite element model validation. Five experienced male university soccer players performed one curve kick. The kicking motions were captured in three-dimensions by two high-speed cameras at 2,500 fps. A finite-element foot model was developed using the barefoot and shoe shape data (Figure 1a). The barefoot, joint, shoe upper, and shoe outsole were assigned linear elastic material properties (Table 1). A finite-element soccer ball model consisted of composite shell elements to form the outer panels and the internal latex bladder layers (Figure 1b). A hyperelastic material model was developed for each layer (Table 1). The foot model was placed in the foot posture immediately before impact in the experiment (Figure 2, Table 2). Finite-element impact analysis was performed for each subject.

Figure 3 a shows the state of contact between the foot and the ball in the finite element analysis for Subject A as a typical example. The impact time for the finite element analysis of the trials for all subjects ranged from 10.0 to $12.0 \mathrm{~ms}$, which was consistent with the impact time of 9.6-12.0 ms measured using the high-speed camera footage. Figure $3 \mathrm{c}$ shows the trajectory of the foot and the ball during impact in the experiment and the finite element analysis for Subject A as a typical example. The coordinate system corresponds to the global coordinate system shown in Figure 2. Each dot in the figure represents the position of each point immediately before impact, and the centre of the ball immediately before impact is positioned at the origin. The trajectory during impact is not among the items to evaluate the validity of the model, but the trajectory of each point of the foot and centre of the ball in the finite element analysis was in good qualitative agreement with the trajectory in the experiment. The points on the foot and the centre of the ball did not exhibit any substantial changes throughout the impact in terms of the accuracy of the trajectory in the finite element analysis. The trajectory in the finite element analysis was also in good agreement with that observed in the experiment for other subjects.

Table 3 shows the results of the launch angle of the ball, ball velocity, and ball rotation immediately after impact in both the experiment and the finite element analysis. The launch angle is the angle in the left/right and up/down directions to the vector of the foot (centre of mass: COM) velocity immediately before impact ( $Y$ direction). The $X, Y$, and $Z$ components of the ball velocity and ball rotation correspond to the global coordinate system shown in Figure 2. The $Y$ component tended to be relatively less accurate for the ball velocity immediately after impact in the finite element analysis. Rotation about the $Y$ axis was also relatively less accurate for the ball rotation. However, the launch angle of the ball, ball velocity, and ball rotation immediately after impact in the finite element analysis were all in general agreement with the experimental results, with the differences from the experimental results varying from $-0.9^{\circ}$ to $1.0^{\circ}$, -0.8 to $0.6 \mathrm{~m} / \mathrm{s}$, and -1.0 to $0.9 \mathrm{rps}$, respectively.

Ball behaviour simulation. Figure 4 and Supplementary Figure S1 show the results of simulated ball behaviour (launch angle, ball velocity, and ball rotation) for the curve kicks of the five subjects by varying the foot velocity immediately before impact, the Young's modulus of the shoe upper, and the coefficient of friction between the shoe upper and the ball. When we simulated the ball behaviour by varying the foot velocity immediately before impact in increments of $1.0 \mathrm{~m} / \mathrm{s}$ in the range of the actual experimental value $\pm 2.0 \mathrm{~m} / \mathrm{s}$ for each subject, the foot velocity immediately before impact had little effect on the launch angle of the ball. However, for all subjects, the larger the foot velocity immediately before impact, the larger the ball velocity, and this effect was large. Furthermore, the larger the foot velocity immediately before impact, the larger the ball rotation for four of the five subjects. Only for Subject E, the ball rotation hardly changed when the foot velocity immediately before impact was varied.

Using a Young's modulus of $10 \mathrm{MPa}$ (equivalent to leather) for the shoe upper in the validated finite element model as the base, we simulated the ball behaviour by varying the Young's modulus of the shoe upper between $5 \mathrm{MPa}$ (equivalent to soft polyurethane) a

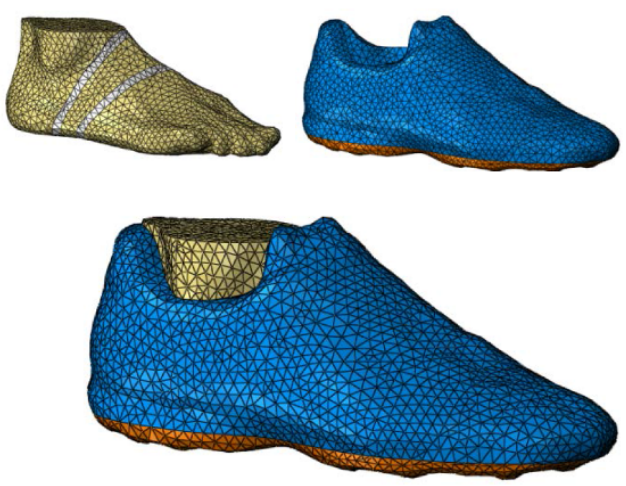

b

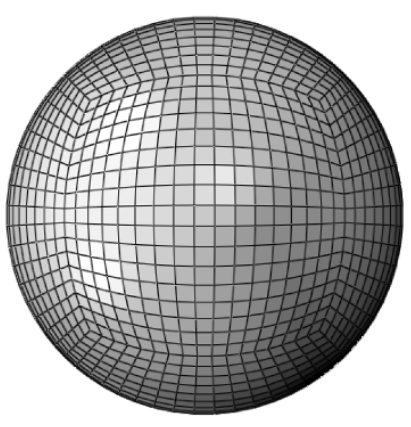

Figure $1 \mid$ Finite element models. (a) A coupled barefoot-shoe model was developed using barefoot and shoe shape data. The tarso-metatarsal and transverse tarsal joints were simulated to model the flexibility of the joint structures. The barefoot, joint, shoe upper, and shoe outsole were assigned linear elastic material properties. (b) The soccer ball model consisted of composite shell elements to form the outer panels and the internal latex bladder layers. A hyperelastic material model was developed for each layer. 
Table $1 \mid$ Material properties of the finite element models

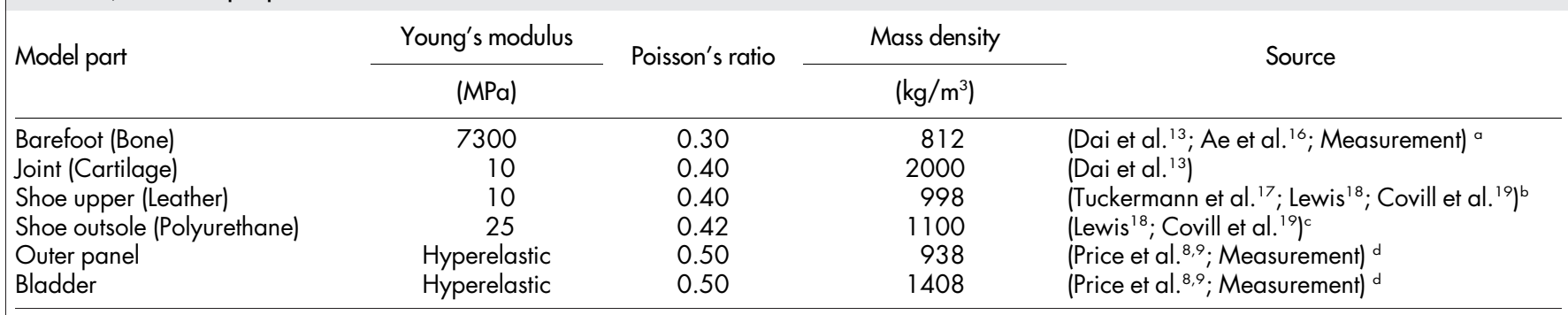

a Young's modulus and Poisson's ratio were obtained from Dai et al. ${ }^{13}$. Mass density was calculated from the mass derived based on Ae et al. ${ }^{16}$ and a volume measurement.

bYoung's modulus was obtained from Tuckermann et al. ${ }^{17}$. Poisson's ratio was obtained from Lewis ${ }^{18}$. Mass density was obtained from Covill et al. ${ }^{19}$.

'Young's modulus and Poisson's ratio were obtained from Lewis ${ }^{18}$. Mass density was obtained from Covill et al. ${ }^{19}$.

'Hyperelastic reduced polynomial strain energy potential equation was fitted against uniaxial tensile test data taken from Price et al. ${ }^{8}$. Poisson's ratio was obtained from Price et al. ${ }^{9}$. Mass density was calculated from the mass and thickness measurements.

and $50 \mathrm{MPa}$ (equivalent to rigid polyurethane) in five increments (5, $10,20,30$, and $50 \mathrm{MPa}$ ). Based on the results, the Young's modulus of the shoe upper had little effect on the launch angle, ball velocity, and ball rotation for all subjects.

Using a coefficient of friction of 0.4 in the validated finite element model as the base, we simulated the ball behaviour by varying the coefficient from 0.2 to 0.6 in increments of 0.1 . According to the results, for four of the five subjects, the coefficient of friction had little effect on the launch angle, ball velocity, and ball rotation. For Subject D, although the coefficient of friction had little effect on the launch angle, ball velocity, and ball rotation in the range of 0.3 to 0.6 (similar to other subjects), when the coefficient of friction was 0.2 , the ball velocity and ball rotation decreased with the changes in the launch angle.

Furthermore, Figure 5 shows the simulation results of the ball velocity and ball rotation by varying the coefficient of friction between the shoe upper and the ball when the foot velocity immediately before impact was larger or smaller $(25.0 \mathrm{~m} / \mathrm{s}$ and $5.0 \mathrm{~m} / \mathrm{s}$, respectively) than each subject's actual experimental value. For all subjects, when the foot velocity immediately before impact was larger $(25.0 \mathrm{~m} / \mathrm{s})$ than the actual experimental value, the effects of the coefficient of friction on ball velocity and ball rotation were similar to its effects when the foot velocity immediately before impact was equal to the actual experimental value (Figures $4 \mathrm{e}$ and $4 \mathrm{f}$ ). In other words, the coefficient of friction had very little effect, except for Subject D when the coefficient of friction was 0.2. On the other hand, when the foot velocity immediately before impact was smaller $(5.0 \mathrm{~m} / \mathrm{s})$ than the actual experimental value, for Subject $\mathrm{D}$, the ball velocity decreased when the coefficient of friction was between 0.2 and 0.3 . Additionally, for Subjects A, B, and D, when the foot velocity immediately before impact was smaller $(5.0 \mathrm{~m} / \mathrm{s})$ than the actual experimental value, the ball rotation tended to decrease when the coefficient of friction decreased. However, for Subjects C and E, the ball rotation hardly changed, even when the coefficient of friction was changed.

\section{Discussion}

The animation of the finite element analysis showed the contact area of the foot press into the ball, deforming the ball at the contact area with the foot. This was similar to the footage from the experiment. The launch of the ball in the left and upward direction to the vector of the foot velocity immediately before impact ( $Y$ direction) and the generation of ball rotation were also observed.

The trajectory during impact in the finite element analysis was in good agreement qualitatively with that observed in the experiment. Nonetheless, this study assumed the joint reaction force and joint moment loaded on the ankle joint to match the trajectory of the heel, lateral malleolus, and three nodes on the lateral side of the foot in the finite element analysis with the trajectory of the markers in the experiment. As a result, the trajectory during impact is not consid- ered for evaluating the validity of the model. This study also used a general shape rather than a specific shape of the subjects for the foot shape in the finite element model. Therefore, the positions of the output nodes on the foot model in the finite element analysis did not perfectly match the marker positions on the subjects in the experiment.

The launch angle, velocity, and rotation of the ball immediately after impact in the finite element analysis were in general agreement with the experimental results. The differences in the results, including the relatively less accurate $Y$ component of the ball velocity and its rotation about the $Y$ axis in the finite element analysis, were due to the simplified inner structure of the finite element foot model, use of constant values for joint reaction force and joint moment loaded on the ankle joint, use of a general shape for the foot shape, and possible errors in the experimental measurements.

The finite element foot model developed in this study is more complex than that used by Asai et al. ${ }^{5}$ who investigated the phenomena occurring during impact and the subsequent ball behaviour for a curve kick, but it is simpler than those used by Chen et al. ${ }^{14}$ and García-González et al. ${ }^{15}$ who investigated the plantar pressure distribution and stress distribution on the bones during contact with the ground; the current finite element foot model did not consider in detail the inner structure of the foot such as bone shape and ligaments. The finite element ball model is also simpler than the ball model used by Price et al. ${ }^{8-11}$ who investigated ball deformation behaviour and stress distribution during a collision against a rigid surface, and did not consider other factors such as the stitching seam between the panels. In the present study, both the foot and the ball models were simplified, but the ball behaviour (launch angle, ball velocity, and ball rotation) in the finite element analysis accurately represented the $3 \mathrm{D}$ properties in the experiment. While the subjects showed diverse impact patterns, as shown in Figure $2 \mathrm{~b}$ and Table 2, the ball behaviour for all subjects in the finite element analysis were simulated well in three dimensions, suggesting that the approach in this study is suitable for diverse impact patterns. The finite element model was deemed valid for simulating the relationship between the impact and the ball behaviour for a curve kick.

The results of simulating the ball behaviour caused by the impact for a curve kick indicate that in all subjects, the larger the foot velocity immediately before impact, the larger the ball velocity. This is similar to the results of previous studies that examined the factors that affect ball velocity for instep kicks ${ }^{3}$ and side-foot kicks ${ }^{4}$ based on numerical calculations using theoretical equations. The larger ball velocity when the foot velocity immediately before impact is large is attributable to the large impact force from the foot that hits the ball. Furthermore, for four of the five subjects, the ball rotation increased along with the ball velocity when the foot velocity immediately before impact was large. From this, it can be inferred that when the foot velocity immediately before impact is large, both the normal and the 
a
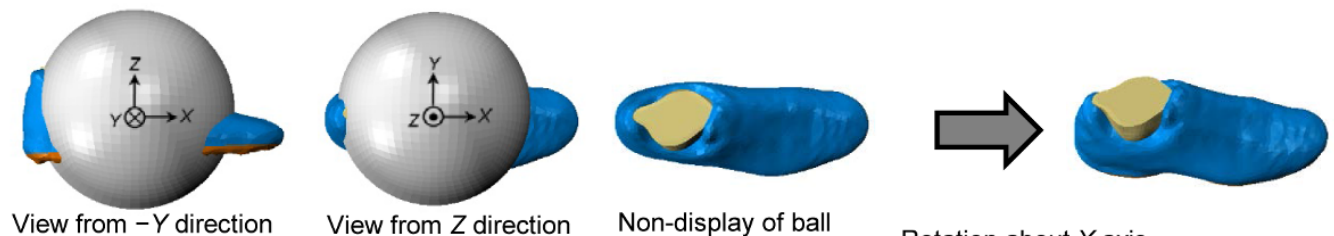

View from $Z$ direction

Non-display of ball

Rotation about $X$ axis

Alignment of the foot local coordinate system to the global coordinate system

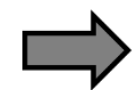

Rotation about $Z$ axis

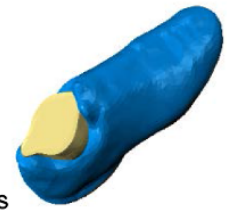

Rotation about $Y$ axis

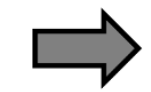

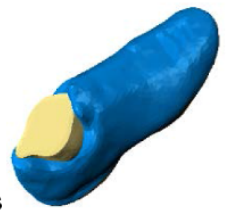
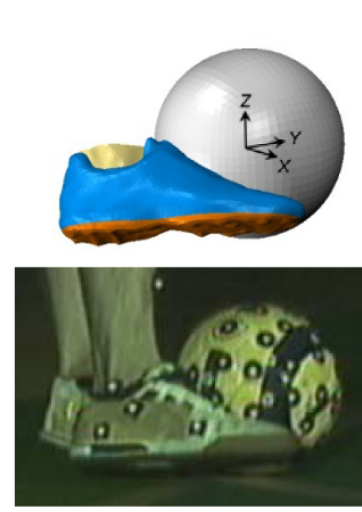

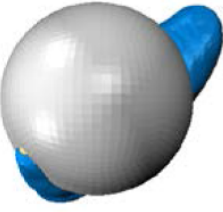

Re-display of ball

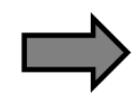

Translation

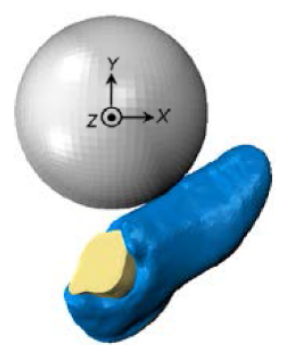

b

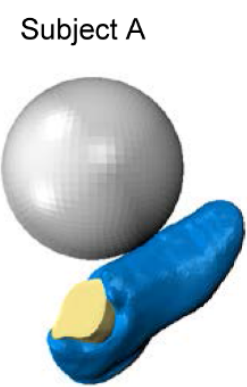

View from $Z$ direction

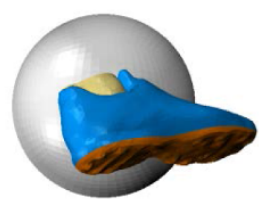

View from $-Y$ direction
Subject B

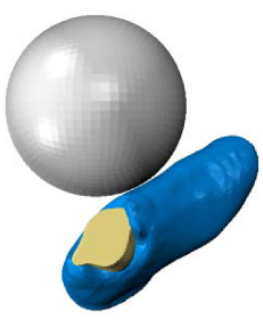

Subject C
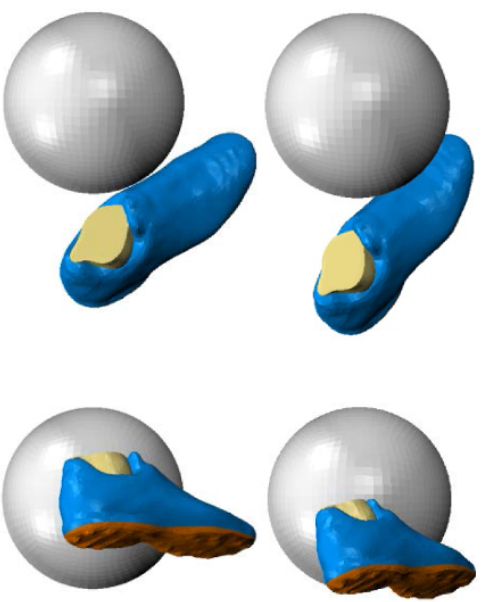

Subject D
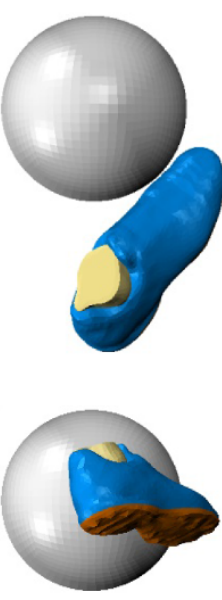

Subject $E$
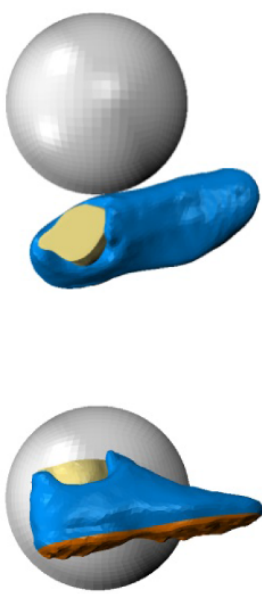

Figure $2 \mid$ Placement of finite element models. (a) A foot model was placed in the foot posture immediately before impact in the experiment. Rotational angles and translational displacements of the foot model for each process are shown in Table 2. Origin of the global coordinate system is located at the centre of the ball immediately before impact. (b) Foot postures immediately before impact viewed from $Z$ and $-Y$ directions for all of the subjects. Impact patterns by the subjects were diverse. As the initial condition, the foot COM velocity immediately before impact in the experiment was applied in the $Y$ direction to the foot model.

tangential component of the impact force from the foot increase. Only for Subject E, the ball rotation hardly changed, even when the foot velocity immediately before impact changed. In the impact for Subject E, because the angle of attack - the angle between the foot velocity vector immediately before impact ( $Y$ direction) and the normal vector to the contact surface of the foot immediately before impact-is small (Figure 2b), the change in the tangential force is smaller even if the foot velocity immediately before impact changes. As a result, it can be inferred that the change in the ball rotation was also small.

Even when the Young's modulus of the shoe upper was varied from $5 \mathrm{MPa}$ (equivalent to soft polyurethane) to $50 \mathrm{MPa}$ (equivalent 
Table 2 | Rotational angles and translational displacements in the placement of the finite element foot model

\begin{tabular}{lrrrrrrrr} 
Subject ID & \multicolumn{3}{c}{ Rotation ( } & & \multicolumn{3}{c}{ Translation (mm) } \\
\cline { 2 - 3 } \cline { 7 - 8 } & \multicolumn{1}{c}{$X$} & $Y$ & $Z$ & & $X$ & $Y$ & \multicolumn{1}{c}{$Z$} \\
\hline A & -25.8 & -1.3 & 45.0 & & 38.7 & -142.6 & -22.2 \\
B & -23.0 & 9.0 & 40.7 & & 52.2 & -149.7 & -14.5 \\
C & -24.1 & 2.6 & 54.5 & & 19.8 & -163.8 & -57.9 \\
D & -33.6 & 11.2 & 55.9 & & 42.3 & -178.3 & 7.3 \\
E & -10.7 & -2.3 & 20.8 & & 25.6 & -156.6 & -14.8 \\
\hline
\end{tabular}

to rigid polyurethane), it had little effect on the launch angle, ball velocity, and ball rotation for all subjects. The shoe upper is just a few millimetres thick and the foot (bone) inside the shoes is much harder than the shoe upper at the impact area. Thus, even when the Young's modulus (hardness) of the shoe upper is varied, its effect on the impact force from the foot is small; as a result, it is presumed to have little effect on the ball behaviour.

Even when the coefficient of friction between the shoe upper and the ball was varied between 0.2 and 0.6 , it had little effect on the launch angle, ball velocity, and ball rotation for four of the five subjects. Although these results are similar to those of Asai et al. ${ }^{5}$, who used a simpler finite element model, the effect on the ball rotation in this study was smaller. In all cases with a coefficient of friction between 0.2 and 0.6 , it is assumed that the ball did not slide on the foot surface, and the contact surface of the ball that touched the foot moved with foot tangential velocity in the tangential direction (i.e., the ball rolled). Thus, even when the coefficient of friction was varied, the ball behaviour, including ball rotation, did not change. This suggested that the tangential force did not reach the maximum static friction force for all cases with a coefficient of friction from 0.2 to 0.6 for these four subjects. When the coefficient of friction was 0.2 , the ball velocity and ball rotation decreased only for Subject D. In the impact of Subject D, because the angle of attack was large (Figure 2b), the tangential velocity of the foot (and the tangential force) was large; with a small coefficient of friction such as 0.2 , it is assumed that the tangential force reaches the maximum static friction force and the ball slides on the foot surface. It is considered that the ball velocity and ball rotation decreased for this reason. The sliding of the ball on the foot surface was also observed in the animation of the finite element analysis for Subject D when the coefficient of friction was 0.2 .

For all subjects, when the foot velocity immediately before impact was equal to the actual experimental value and when it was larger than the actual experimental value, i.e., $25 \mathrm{~m} / \mathrm{s}$, there was hardly any change in the ball velocity and ball rotation, even when the coefficient of friction changed between the shoe upper and the ball. This sug- a

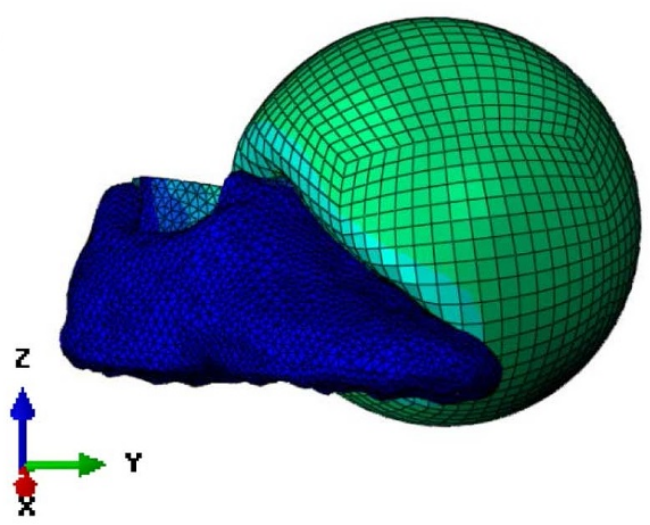

b

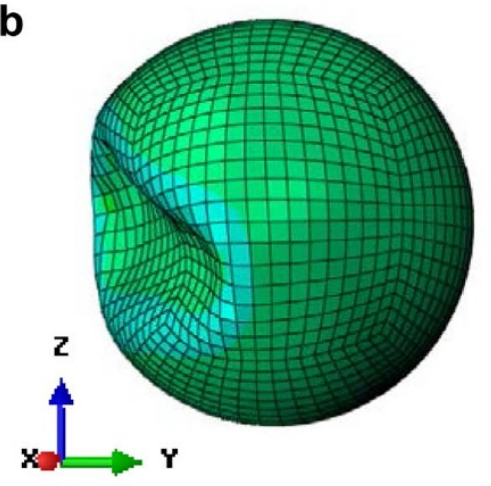

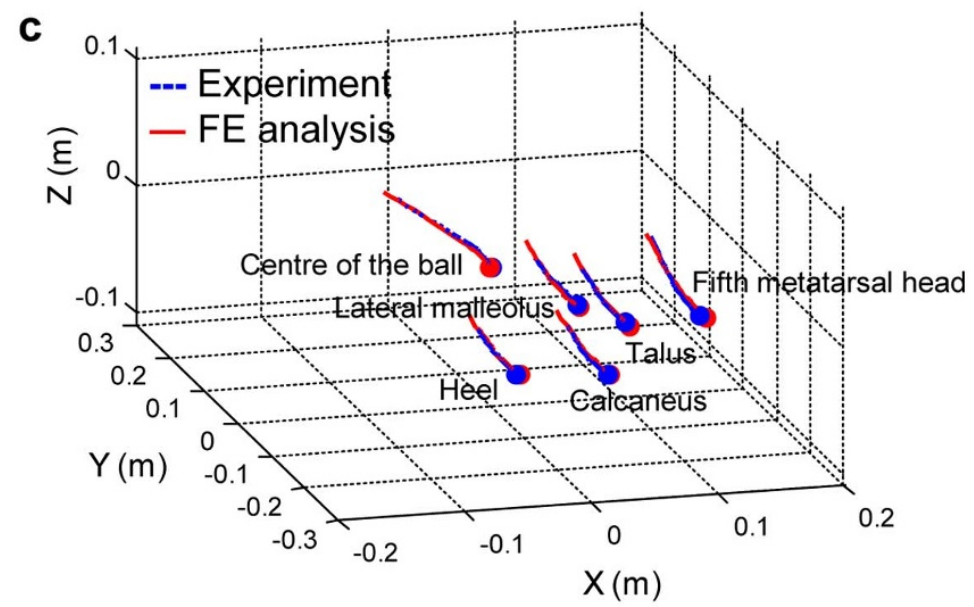

Figure 3 Impact of curve kicks in a finite element analysis for Subject A. (a) Contact of the foot with the ball and (b) deformation shape of the ball. Contact area of the foot pressed into the ball, deforming the ball at the area in contact with the foot. (c) Trajectory of the foot and the ball during impact in the experiment and finite element analysis. Coordinate system corresponds to the global coordinate system shown in Figure 2. Each dot represents the position of each point immediately before impact, and the centre of the ball immediately before impact is positioned at the origin. 
Table 3 | Comparison of the experimental and finite element results for ball behaviour immediately after impact

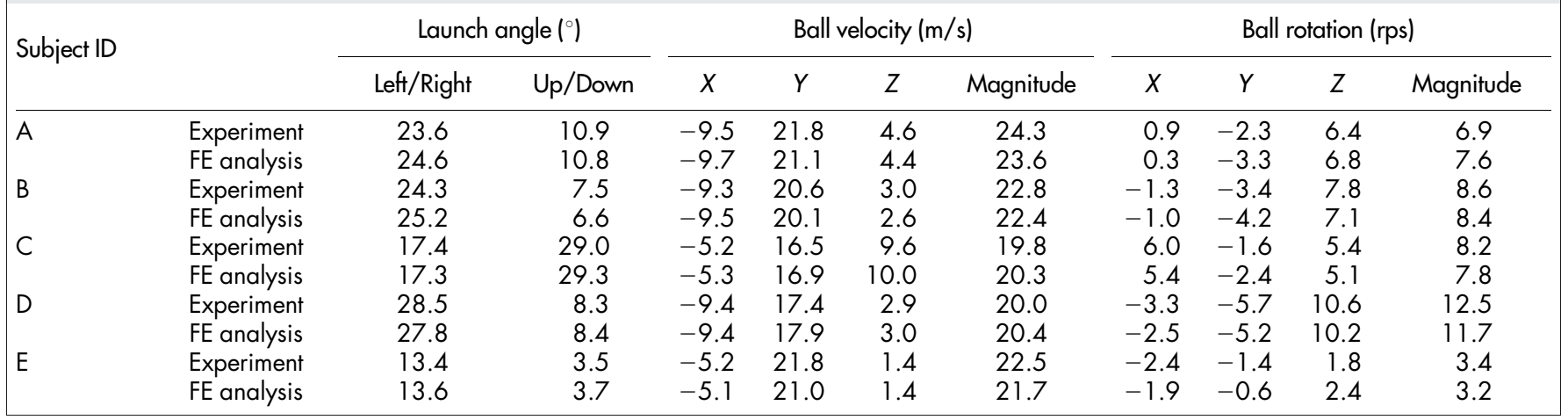

gested that the coefficient of friction did not affect the ball velocity and ball rotation for free kicks and strong shots. On the other hand, when the foot velocity immediately before impact was smaller than the actual experimental value, i.e., $5 \mathrm{~m} / \mathrm{s}$, for Subjects A, B, and D, the ball rotation tended to decrease when the coefficient of friction decreased. This suggested that the coefficient of friction may affect the ball rotation that occurs during dribbling and trapping. However, the ball velocity decreased when the coefficient of friction was 0.2 to 0.3 for Subject $\mathrm{D}$ alone, whereas minimal changes were observed for Subjects A and B. Because the tangential force is smaller than the normal force, the change in the magnitude of the impact force (resultant force of the normal and tangential forces) is small even when only the tangential force changes. Therefore, it is assumed that it is more difficult to observe changes in the ball velocity than in the ball rotation. For Subjects C and E, the ball rotation was initially lower than $3.0 \mathrm{rps}$ when the foot velocity immediately before impact was $5 \mathrm{~m} / \mathrm{s}$, which was smaller than the actual experimental value. It is presumed that because the change in tangential force was small even when the coefficient of friction changed, the change in ball rotation was also small. When the foot velocity immediately before impact was small, the ball rotation tended to decrease when the coefficient of friction decreased. However, this tendency was not observed when the foot velocity immediately before impact was large. It is presumed that because a large deformation of the ball occurs during the impact in this case, the foot presses into the ball, which enters a contact state in which it is difficult to slide even if the coefficient of friction is small.

One major limitation of the model developed in this study is that the inner structure of the foot was simplified. As a result, this model cannot be used in studies that attempt to investigate the stress distribution on the barefoot region during impact. For the same reason,

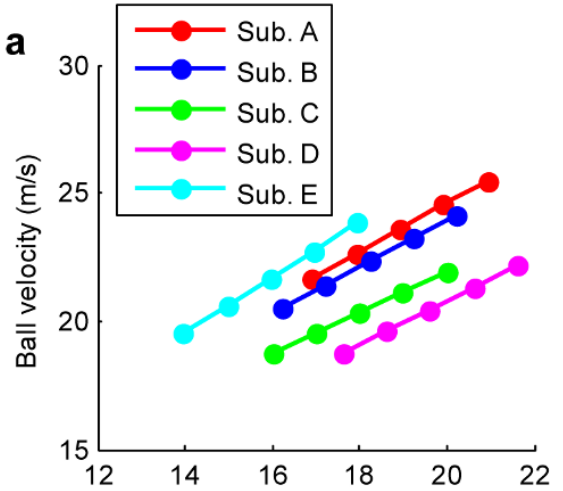

Foot velocity immediately before impact $(\mathrm{m} / \mathrm{s})$

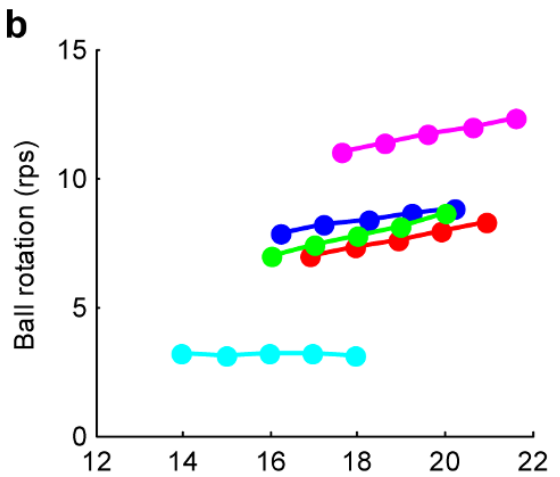

Foot velocity immediately before impact $(\mathrm{m} / \mathrm{s})$

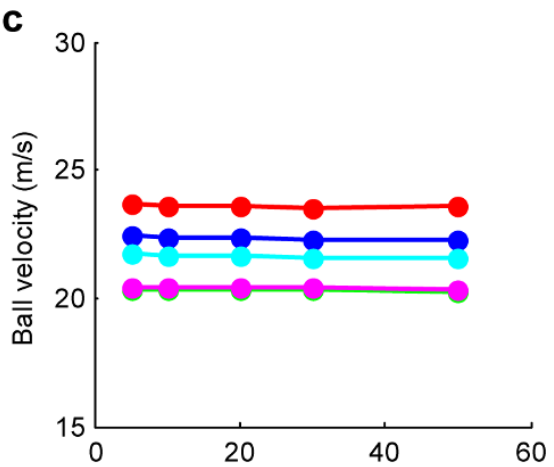

Young's modulus of shoe upper (MPa)

d

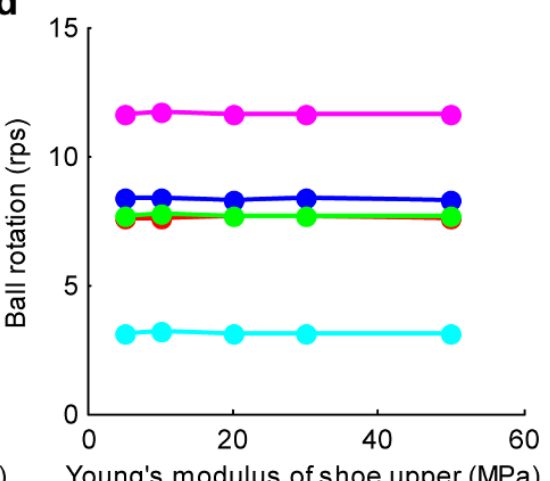

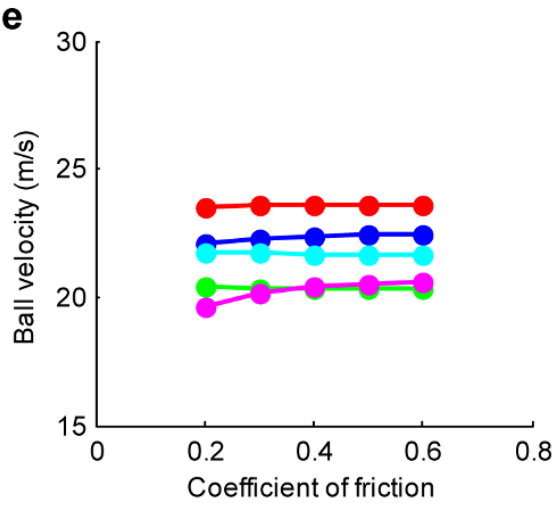

f

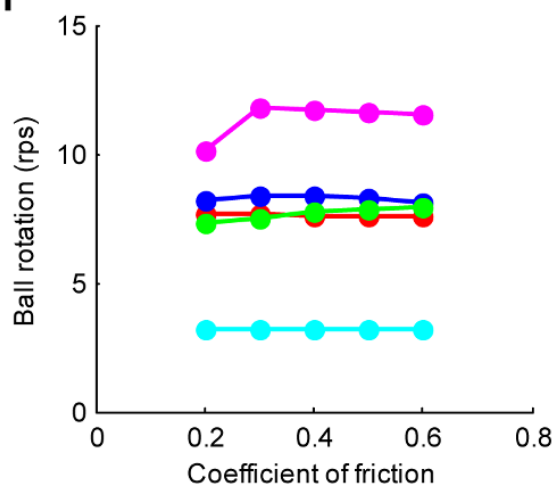

Figure 4 Simulation results of ball velocity and ball rotation for the curve kicks of five subjects by varying (a and b) the foot velocity immediately before impact, ( $c$ and d) the Young's modulus of the shoe upper, and (e and f) the coefficient of friction between the shoe upper and the ball. The results of launch angles in the left/right and up/down directions to the vector of the foot velocity immediately before impact, $X, Y$, and $Z$ components of the ball velocity and ball rotation are shown in Supplementary Figure S1. 

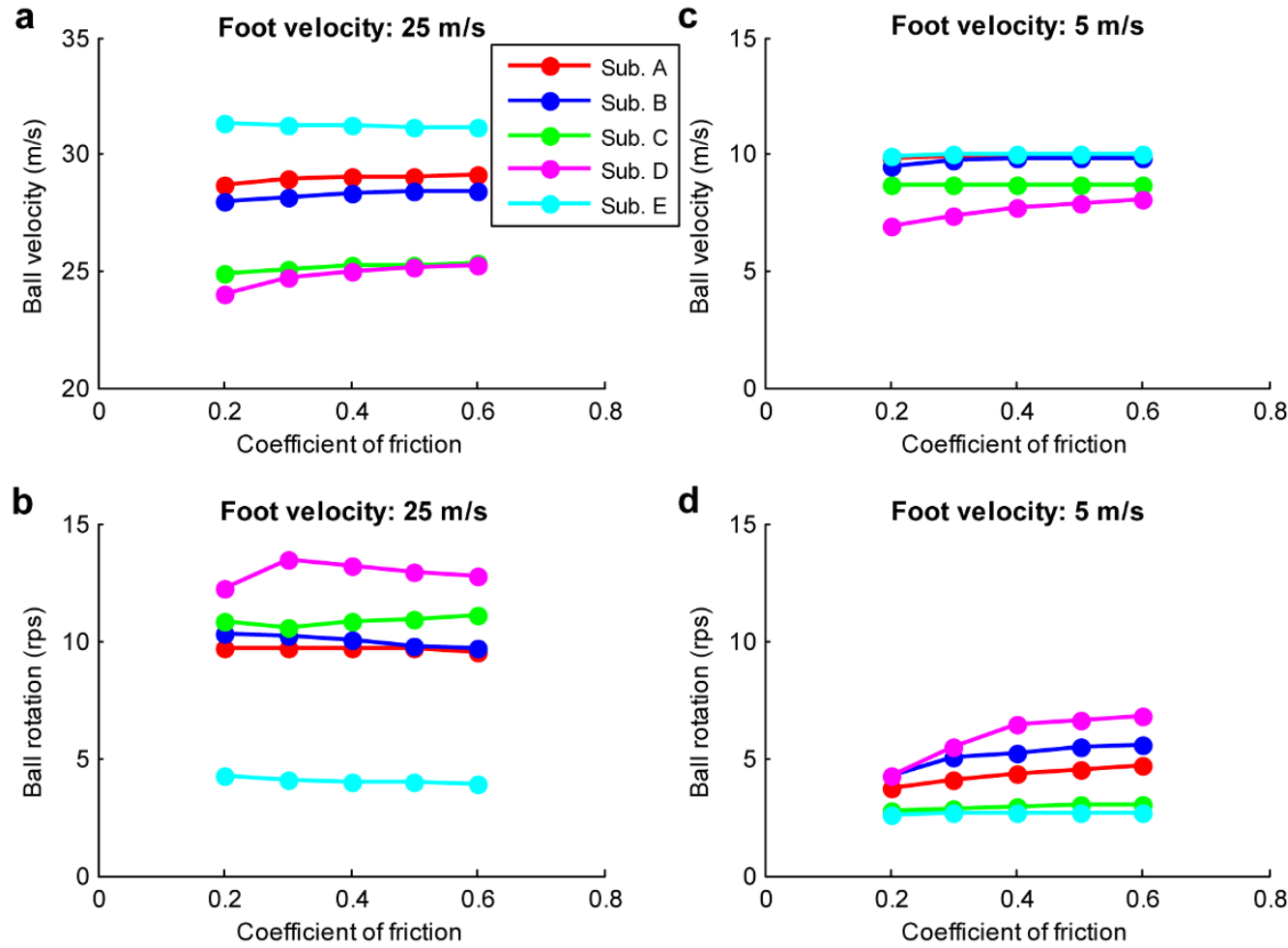

Figure 5 Simulation results of ball velocity and ball rotation for five subjects by varying the coefficient of friction between the shoe upper and the ball when the foot velocity immediately before impact was ( $a$ and b) larger or (c and d) smaller $(25.0 \mathrm{~m} / \mathrm{s}$ and $5.0 \mathrm{~m} / \mathrm{s}$, respectively) than each subject's actual experimental value.

this model is unsuitable for investigating the plantar pressure distribution while in contact with the ground during walking or running. Finally, this model cannot simulate the relationship between the impact and the ball behaviour for toe kicks, which causes great deformation in the toe portion of the foot. The other major limitation of this model is that the joint reaction force and joint moment loaded on the ankle joint were constant values. Although the constant values were used by reference to the previous studies ${ }^{13-15}$, the ankle joint reaction force and ankle joint moment during impact may not be constant in fact. It is considered that this assumption affects the accuracy of the finite element analysis. However, the launch angle, ball velocity, and ball rotation immediately after impact in the finite element analysis were in general agreement with the experimental results.

Although the results of this study suggest that the shoe upper does not significantly influence the ball behaviour (launch angle, ball velocity, and ball rotation) immediately after kick impact, it cannot be concluded that the shoes do not influence the ball behaviour. Among the parameters related to shoes, we examined the effect of Young's modulus of the shoe upper and the coefficient of friction on ball behaviour. However, other material properties may affect the ball behaviour. Alternatively, attaching thick rubber or another protuberance to the shoe upper to create a 3D structure may influence the ball behaviour. However, we speculate that the influence of the impact pattern on the ball behaviour is much greater than that of the shoe upper. In other words, to kick a curve ball or fast ball as intended, the kicker's technique is of paramount importance. Scoring a goal using these kicks is possible only because of the player's superior skill at the required kick techniques. If the finite element model constructed in this study can be applied to curve kicks with varying impact patterns or to knuckle ball kicks, the relationship between the impact patterns and the ball behaviour can be further understood. These results are likely to contribute to the improvement of the players' skills and improve the development of products such as improved soccer shoes.

\section{Methods}

Experiment. The study included five experienced male university soccer players (age: $22.6 \pm 2.1$ years; height: $172.2 \pm 5.4 \mathrm{~cm}$; and body mass: $59.4 \pm 6.3 \mathrm{~kg}$ ), each with more than 10 years of playing experience. This study was conducted in accordance with the protocol approved by the Research Ethics Committee of the Tokyo Institute of Technology. The subjects provided written informed consent before the experiment. All subjects were right-footed kickers. The experiment was conducted in an indoor laboratory with artificial turf, and the subjects wore shoes designed for artificial turf (size: $25.5-27.0 \mathrm{~cm}$; mass: 240-290 g). A size 5 FIFA-approved soccer ball (mass: $432 \mathrm{~g}$ ) was used, and the inflation pressure was maintained at $9.7 \mathrm{psi}$ throughout the experiment.

The subjects were instructed to perform one curve kick toward a goal that was positioned $4 \mathrm{~m}$ away. They were not given specific directions about impact pattern. The kicking foot motions were captured by two electrically synchronised high-speed cameras (Memrecam fx-K4, NAC Image Technology Inc., Tokyo, Japan) at 2,500 fps (exposure time 1/5,000 s). The cameras were placed to the right lateral and right rear of the subjects.

Full Methods of Experiment and Experimental data processing can be found in the Supplementary Methods online.

Finite element model construction. Effective models are simple and can solve the research problem. The aim of this study was to examine the relationship between the impact and ball behaviour for curve kicks. Because the impact area for a curve kick is a hard region, which consists of bones ranging from the dorsal aspect to the medial aspect of the foot, details of the inner structure of a foot such as each bone shape and the ligaments were assumed to be unnecessary in the finite element model, which was therefore simplified. A generalised foot shape was also assumed to be usable as the shape for the foot model rather than applying a shape that is specific to each subject. First, the barefoot and shoe (shod foot) shapes of another male subject (age: 34 years, height: $170.0 \mathrm{~cm}$, body mass: $64.0 \mathrm{~kg}$ ) were obtained using a 3D foot laser scanner (INFOOT, I-Ware Laboratory Co., Ltd., Osaka, Japan) under static conditions after informed consent was obtained from the subject. When measuring the barefoot shape, six markers with diameters of $5 \mathrm{~mm}$ were placed on anatomical landmarks of the tarso-metatarsal joint (tuberosity of the first metatarsalis, tuberosity of the second metatarsalis, and tuberosity of the fifth metatarsalis) and the transverse tarsal joint (tuberosity of navicular, junction point, and tuberosity of cuboid) to simulate these joints for later modelling of the flexibility of the joint structures.

The barefoot and shoe shape data were then imported into the finite element software application Abaqus/CAE (Version 6.11, Dassault Systèmes, Vélizy-

Villacoublay, France) and were merged while retaining the boundary between the barefoot and the shoe. The finite element foot model was scaled to the foot size of each subject. The foot model was meshed using tetrahedral solid elements (Figure 1a). The barefoot, joint, shoe upper, and shoe outsole were assigned linear elastic material 
properties (Table 1). The 3D joint reaction force and joint moment (constant loads) were applied to the ankle joint centre node.

The ball was modelled as a hollow sphere and was meshed using quadrilateral composite shell elements to form the outer panels ( $2.5 \mathrm{~mm}$ thick) and the internal latex bladder ( $0.4 \mathrm{~mm}$ thick) layers (Figure $1 \mathrm{~b})$. A hyperelastic material model was developed for each part by fitting a hyperelastic reduced polynomial strain energy potential equation against uniaxial tensile test data taken from Price et al. ${ }^{8}$ (Table 1). To describe the viscoelastic material behaviour, a stiffness proportional damping was applied to each composite material layer ${ }^{8}$. Mass densities of the outer panels and bladder were calculated from mass and thickness measurements. A fluid cavity was defined to represent the air within the bladder and allow for pressurisation. The ball inflation pressure used for the experiments was applied to the ball model.

The foot model was placed in the foot posture immediately before impact in the experiment as shown in Figure 2. The rotational angles and translational displacements of the foot model for each process are shown in Table 2. As the initial condition, the foot COM velocity immediately before impact in the experiment was applied in the $Y$ direction to the foot model. The foot angular velocity immediately before impact in the experiment was also applied to the foot model. The friction between the foot model and the ball model was expressed by the Coulomb friction, and the coefficient of friction was set at $0.4^{5}$.

Finite element impact analysis was performed using Abaqus/Explicit, and the coordinates of eight nodes (heel, lateral malleolus, three nodes on the lateral side of the foot, and three nodes on the ball surface) were output at $0.4 \mathrm{~ms}$ intervals corresponding to the frame rate of the high-speed cameras used in the experiment.

The trajectory of the foot and centre of the ball during impact were obtained from both the experiment and finite element analysis. The centre of the ball in the finite element analysis was calculated using the same method as that for the experiment. For comparisons, the experimental data were transformed to a global coordinate system with the origin at the centre of the ball immediately before impact with the $Y$ axis parallel to the vector of the foot COM velocity immediately before impact (the global coordinate system is shown in Figure 2). The values of the joint reaction force and joint moment applied to the ankle joint were determined so that the trajectories of five nodes (heel, lateral malleolus, and three nodes on the lateral side of the foot) in the finite element analysis were in good agreement with the respective marker trajectory in the experiment.

Finite element model validation. The ball behaviour immediately after impact was obtained from both the experiment and finite element analysis (after determination of the values of the ankle joint loads). The launch angles of the ball in the left/right and up/down directions to the vector of the foot (COM) velocity immediately before impact ( $Y$ direction) were computed using the centre of the ball. Each component of the ball velocity immediately after impact was derived from the first derivative of the regression equation fitted to the displacement after impact. The absolute magnitude of the ball velocity vector was calculated from the values of its components. The ball rotation about each axis of the global coordinate system was calculated using the three points on the ball surface. The ball rotation about its own rotational axis (absolute magnitude of the rotation) was calculated from the values of its rotation vector components. The finite element model was validated by comparing the finite element results for the ball behaviour (launch angle, ball velocity and ball rotation) immediately after impact with the experimental results for each trial (each subject).

Ball behaviour simulation. To study the factors that affect the ball behaviour for curve kicks, we used a validated finite element model. For each subject, we simulated the ball behaviour that occurs during the impact by varying the foot velocity immediately before impact, the Young's modulus of the shoe upper, and the coefficient of friction between the shoe upper and the ball. We simulated the ball behaviour by varying the foot velocity immediately before impact in increments of $1.0 \mathrm{~m} / \mathrm{s}$ in the range of the actual experimental value $\pm 2.0 \mathrm{~m} / \mathrm{s}$ for each subject. As the base, we used the Young's modulus of the shoe upper of $10 \mathrm{MPa}$, which is equivalent to that of leather, in the validated finite element model. We simulated the ball behaviour by varying it from $5 \mathrm{MPa}$, which is equivalent to soft polyurethane, to $50 \mathrm{MPa}$, which is equivalent to rigid polyurethane, over five stages $(5,10,20,30$, and $50 \mathrm{MPa}$ ). As the base, we used the coefficient of friction of 0.4 in the validated finite element model; we simulated the ball behaviour by varying it from 0.2 to 0.6 in increments of 0.1 .

Furthermore, to determine the relationship between the foot velocity immediately before impact and the effect of the coefficient of friction between the shoe upper and the ball on the ball velocity and ball rotation, we simulated the ball velocity and ball rotation by varying the coefficient of friction when the foot velocity immediately before impact was larger or smaller $(25.0 \mathrm{~m} / \mathrm{s}$ and $5.0 \mathrm{~m} / \mathrm{s})$ than each subject's actual experimental value.

1. Sterzing, T. \& Hennig, E. M. The influence of soccer shoes on kicking velocity in full-instep kicks. Exerc. Sport Sci. Rev. 36, 91-97 (2008).

2. Andersen, T. B., Dörge, H. C. \& Thomsen, F. I. Collisions in soccer kicking. Sports Eng. 2, 121-125 (1999)
3. Ishii, H., Yanagiya, T., Naito, H., Katamoto, S. \& Maruyama, T. Theoretical study of factors affecting ball velocity in instep soccer kicking. J. Appl. Biomech. 28, 258-270 (2012).

4. Ishii, H., Yanagiya, T., Naito, H., Katamoto, S. \& Maruyama, T. Numerical study of ball behavior in side-foot soccer kick based on impact dynamic theory. J. Biomech. 42, 2712-2720 (2009).

5. Asai, T., Carré, M. J., Akatsuka, T. \& Haake, S. J. The curve kick of a football I: impact with the foot. Sports Eng. 5, 183-192 (2002).

6. Asai, T., Takano, S., Carré, M. J. \& Haake, S. J. A fundamental study of an infront curve kick in football. In: Hubbard, M., Mehta, R. D. \& Pallis, J. M. (eds.) The Engineering of Sport 5, 290-295 (Sheffield: International Sports Engineering Association, 2004).

7. Asai, T., Nunome, H., Maeda, A., Matsubara, S. \& Lake, M. Computer simulation of ball kicking using the finite element skeletal foot model. In: Reilly, T., Cabri, J. \& Araújo, D. (eds.) Science and Football V, 77-82 (London: Routledge, 2005).

8. Price, D. S., Harland, A. R. \& Jones, R. Computational modelling of manually stitched soccer balls. Proc. Inst. Mech. Eng. L 220, 259-268 (2006).

9. Price, D. S., Harland, A. R. \& Jones, R. Soccer ball anisotropy modelling. Mater. Sci. Eng. A Struct. Mater. 420, 100-108 (2006).

10. Price, D. S., Jones, R. \& Harland, A. R. Advanced finite-element modelling of a 32 panel soccer ball. Proc. Inst. Mech. Eng. C 221, 1309-1319 (2007).

11. Price, D. S., Jones, R., Harland, A. R. \& Silberschmidt, V. V. Viscoelasticity of multi-layer textile reinforced polymer composites used in soccer balls. J. Mater. Sci. 43, 2833-2843 (2008).

12. Rezaei, A., Verhelst, R., Van Paepegem, W. \& Degrieck, J. Finite element modelling and experimental study of oblique soccer ball bounce. J. Sports Sci. 29, 1201-1213 (2011).

13. Dai, X. Q., Li, Y., Zhang, M. \& Cheung, J. T. Effect of sock on biomechanical responses of foot during walking. Clin. Biomech. 21, 314-321 (2006).

14. Chen, W. M., Park, J., Park, S. B., Shim, V. P. \& Lee, T. Role of gastrocnemiussoleus muscle in forefoot force transmission at heel rise - A 3D finite element analysis. J. Biomech. 45, 1783-1789 (2012).

15. García-González, A., Bayod, J., Prados-Frutos, J. C., Losa-Iglesias, M., Jules, K. T., Becerro de Bengoa-Vallejo, R. \& Doblaré, M. Finite-element simulation of flexor digitorum longus or flexor digitorum brevis tendon transfer for the treatment of claw toe deformity. J. Biomech. 42, 1697-1704 (2009).

16. Ae, M., Tang, H. \& Yokoi, T. Estimation of inertia properties of the body segments in Japanese athletes. In: Society of Biomechanisms (ed.) Biomechanisms 11, 23-33, (Tokyo: University of Tokyo Press, 1992) (in Japanese with English abstract).

17. Tuckermann, M., Mertig, M., Pompe, W. \& Reich, G. Stress measurements on chrome-tanned leather. J. Mater. Sci. 36, 1789-1799 (2001).

18. Lewis, G. Finite element analysis of a model of a therapeutic shoe: effect of material selection for the outsole. Biomed. Mater. Eng. 13, 75-81 (2003).

19. Covill, D., Guan, Z. W., Bailey, M. \& Raval, H. Development of thermal models of footwear using finite element analysis. Proc. Inst. Mech. Eng. H 225, 268-281 (2011).

\section{Acknowledgments}

The authors gratefully thank NAC Image Technology, Inc., for providing the high-speed cameras used in the experiment. The authors also acknowledge Mitsunori Tada, Katsumasa Tanaka, and Masaaki Mochimaru for their advice on the finite element analysis. This research was supported by Grants-in-Aid for Scientific Research from the Japan Society for the Promotion of Science (KAKENHI 23700744).

\section{Author contributions}

H.I. and T.M. designed the experiments; H.I., Y.S. and T.M. performed the experiments; H.I. analysed the experimental data with help from Y.S. and T.M.; H.I. performed the finite element analysis with help from Y.S.; and H.I. wrote the paper

\section{Additional information}

Supplementary information accompanies this paper at http://www.nature.com/ scientificreports

Competing financial interests: The authors declare no competing financial interests. How to cite this article: Ishii, H., Sakurai, Y. \& Maruyama, T. Effect of soccer shoe upper on ball behaviour in curve kicks. Sci. Rep. 4, 6067; DOI:10.1038/srep06067 (2014).

This work is licensed under a Creative Commons Attribution-NonCommercialNoDerivs 4.0 International License. The images or other third party material in this article are included in the article's Creative Commons license, unless indicated otherwise in the credit line; if the material is not included under the Creative Commons license, users will need to obtain permission from the license holder in order to reproduce the material. To view a copy of this license, visit http:// creativecommons.org/licenses/by-nc-nd/4.0/ 